\title{
ON SERIES INTEGRALS AND CONTINUED FRACTIONS - I
}

\author{
By K. RAMACHANDRA
}

I To my Teachers at School and College on the occasion of the EIGHTIETH BIRTHDAY of Professor B. S. MADHAVA RAO, D.Sc., F.A.,Sc., F.N.I., Retired Principal, Central College, Bangalore)

\section{§ I. Introduction}

I had not thought of publishing my researches in my school and college days. Now I believe that a few of them deserve to be printed. One of them is the evaluation

$$
\begin{gathered}
{ }_{z}^{1}\left(\frac{z}{z+1}\right)^{2}+\frac{1}{z+2}\left(\frac{z \cdot z+2}{z+1 \cdot z+3}\right)^{2}+ \\
\frac{1}{z+4}\left(\frac{z . z+2 . z+4}{z+1 \cdot z+3 . z+5}\right)^{2}+\ldots \ldots . \\
=z-\frac{\pi}{2 z-3} \frac{r^{2}(z)}{\left(r\left(\frac{z}{2}\right)\right)^{4}} .
\end{gathered}
$$

My earlier proof of this depended on relating the series to a suitable continued fraction $a+\frac{b_{1}}{a}+\frac{b_{2}}{a}+\frac{b_{3}}{a}+\ldots$ and then evaluating the latter. The evaluation of this continued fraction was done independently by $m e$ in an attempt to prove formula (1.8) (page 8 of [2]) of S. Ramanujan. But (1) is related to the continued fraction whose evaluation seems to be known to 
L. Euler; see [1]. I have now forgotten my earlier proof of (1). I give another proof which lends itself to further generalizations. These results seem to be new. As a corollary to our method many results follow of which I state one at once.

\section{Theorem I}

Let $a_{i}, \beta_{i}(i=1$ to $k)$ be complex constants and $z$ a comple variable. Let $\Sigma \beta_{i}-1+\Sigma a_{i}$ and $\phi(z)$

$$
=\prod_{i-1}^{k}\left(\frac{z+a_{i}}{z+\beta_{i}}\right), \lambda(z)=-1-z+z(\phi(z))^{-1} \text {. Then, we }
$$

have, $\lambda(z) \phi(z)+\lambda(z+1) \phi(z) \phi(z+1)+\lambda(z+2)$

$\phi(z) \phi(z+1) \phi(z+2)+\ldots=z-\prod_{i=1}^{k} \frac{\Gamma\left(z+\beta_{i}\right)}{\Gamma\left(z+a_{i}\right)}$.

for all values of the parameters for which both sides make sense. Also, we have a trivial summation,

$b_{0}=\left(\frac{b_{0}}{a_{0}}-b_{1}\right) a_{0}+\left(\frac{b_{1}}{a_{1}}-b_{2}\right) a_{0} a_{1}+\left(\frac{b_{2}}{a_{2}}-b_{3}\right) a_{0} a_{1} a_{2}$ $+\ldots \ldots$

Hence

$$
\begin{aligned}
1= & \left(\frac{1}{\phi(z)}-1\right) \phi(z)+\left(\frac{1}{\phi(z+1)}-1\right) \phi(z) \phi(z+1) \\
& +\left(\frac{1}{\phi(z+2)}-1\right) \phi(z) \phi(z+1) \phi(z+2)+\cdots
\end{aligned}
$$

Remark 1

If we put $k=2, a_{1}=a_{2}=0, \beta_{1}=\beta_{2}=1$ in (2) we get $\phi(z)=\left(\frac{2 z}{2 z+1}\right)^{2}$ and $\lambda(z)-1-z+z^{-1}(z+1)^{2}$ $=\frac{1}{4 z}$. If we now write $z / 2$ in place of $z$ and use the dupli- 
cation formula for $\Gamma(2 z)$ we get (1), We record the special case $z=1$ of (1). namely

$$
{ }_{1}^{1}\left(\frac{1}{2}\right)^{2}+\frac{1}{3}\left(\frac{1.3}{2.4}\right)^{2}+\frac{1}{5}\left(\frac{1.3 .5}{2.4 .6}\right)^{2}+\ldots=1-\frac{2}{\pi} .
$$

Note that $\frac{1}{\phi(z)}-1=\frac{1}{z}+\frac{1}{(2 z)^{2}}$ and so from (4) with $z=1$ we get (on using the expression for $1-\frac{2}{\pi}$ )

$$
\frac{1}{1^{2}}\left(\frac{1}{2}\right)^{2}+\frac{1}{3^{2}}\left(\frac{1.3}{2.4}\right)^{2}+\frac{1}{5^{2}}\left(\frac{1.3 .5}{2.4 .6}\right)^{2}+\ldots=\frac{4}{\pi}-1 .
$$

But these are not new. The second was first discovered by Euler [1].

Remark 2. If in (2) we put $k=2 m, a_{1}=\ldots=a_{m}=\frac{1}{2}$, $a_{m+1}=\ldots=a_{2 m}=-\frac{1}{b}, \beta_{1}=\beta_{2}=\ldots=\beta_{2 m-1}=0$, $\beta_{2 m}=1$, we get $\phi(z)=\left(z^{2}-\frac{1}{4}\right)^{m} z^{1-2 m}(z+1)^{-1}$ and $\begin{aligned} \lambda(z) & =-1-z+(z+1) z^{2 m}\left(z^{2}-\frac{1}{4}\right) \\ & =\frac{z^{2 m}(z+1)}{\left(z^{2}-\frac{1}{4}\right)^{m}}\left(1-\left(1-\frac{1}{4 z^{2}}\right)^{m}\right), \text { and }\end{aligned}$ $\phi z) \phi(z+1) \ldots \phi(z+n)$

$$
\begin{aligned}
=\left(\frac{\left(z-\frac{1}{2}\right)}{z(z+1) \ldots(z+n)}\right)^{2 m} & \\
& \quad \times\left(\frac{z\left(z+\frac{1}{2}+n\right)}{(z+n+1)\left(z-\frac{1}{2}\right)^{m}}\right) .
\end{aligned}
$$

Remark 3. By linear combinations of (2) and (4) we can get some nice formulae for $\left(\frac{1}{\pi}\right)^{m}(m=1,2,3, \ldots)$ which are, I believe, new. For this purpose a convenient choice is $k=2 m$, $a_{i}=0(i=1$ to $k), \beta_{k}=\beta_{k-1}=1, \beta_{1}=\beta_{2}=\ldots$ 
$-\boldsymbol{\beta}_{m-1}=\mathbf{t}=-\boldsymbol{\beta}_{m}=-\boldsymbol{\beta}_{m-1}=\ldots=-\boldsymbol{\beta}_{k}-2$. Hence $\phi(z)=z^{2 m}\left((z+1)^{m+1}\left(z-\frac{1}{2}\right)^{m-1}\right)^{-1}$, $\frac{1}{\phi(z)}-1=\left(1+\frac{1}{2 z}\right)^{m+1}\left(1-\frac{1}{2 z}\right)^{m-1}-1$ $=\left(1+\frac{1}{z}+\frac{1}{4 z^{2}}\right)_{\gamma=0}^{m-1}\left(\begin{array}{c}m-1 \\ \gamma\end{array}\right)\left(\frac{-1}{4 z^{2}}\right)^{\gamma}-1$, $\lambda(z)=-1+z\left(\frac{1}{\phi(z)}-1\right)$

$$
=\frac{1}{4 z}+z\left(1+\frac{1}{2 z}\right)^{2} \underset{\gamma=1}{m-1}\left(\begin{array}{c}
m \\
\gamma
\end{array}\right)\left(-\frac{1}{4 z^{2}}\right)^{\gamma},
$$

$\phi(z) \phi(z+1) \ldots \phi(z+n)$

$=\left(\frac{z(z+1) \ldots(z+n)}{\left(z+\frac{1}{2}\right) \ldots\left(z+\frac{1}{2}+n\right)}\right)^{2 m}\left(\frac{z+n+\frac{1}{2}}{z-1}\right)^{m-1}$.

With this choice we get the formula

$z-\left(\frac{\Gamma\left(z+\frac{1}{2}\right) \Gamma(z-1)}{(\Gamma(z))^{2}}\right)^{m}\left(\begin{array}{l}\Gamma\left(z+\frac{1}{b}\right) \\ \Gamma\left(z-\frac{1}{2}\right)\end{array}\right)=$

$\gamma=\sum_{=0}^{\infty} \lambda(z+\gamma) \phi(z) \phi(z+1) \ldots \phi(z+\gamma) \ldots$

Putting $z=3 / 2$ we get $\phi\left(\frac{3}{2}\right) \phi\left(\frac{5}{2}\right) \ldots \phi\left(\frac{3}{2}+n\right)$

$=\left(\frac{3 \ldots(2 n+3)}{4.6 \ldots(2 n+4)}\right)^{2 m}\left(\frac{n+2}{n+1}\right)^{m-1}$,

$\frac{3}{2}-\left(\frac{4}{\pi}\right)^{m}=2^{2 m} \sum_{n=0}^{\infty} \psi_{m}(n)\left(\begin{array}{c}1.3 \ldots 2 n+3 \\ 2.4 \ldots 2 n+4\end{array}\right)^{2 m}$, where

$$
\begin{array}{r}
\psi_{m}(n)=\frac{1}{4 n+6}\left(\frac{n+2}{n+1}\right)^{m-1}\left[1+(2 \mathrm{n}+4)^{2}\right. \\
\left.\underset{\gamma=1}{m-1} \sum_{\gamma}^{m-1},\left(\frac{-1}{(2 n+3)^{2}}\right)^{\gamma}\right]
\end{array}
$$


Remark 4. It is possible to generalise theorem 1 further by relaxing the restrictions like $\Sigma \beta_{i}=1+\Sigma a_{i}$. This will be apparent on examining our proof of theorem 1 . However we reserve these for another paper.

\section{§ 2. Proof of Theorem I}

The proof can be split up into a few easy lemmas.

Lemma 1 A solution of the equation

$$
\psi(z)=\lambda(z) \phi(z)+\phi(z) \psi(z+1) \text { is } \psi(z)=z .
$$

Proof: We choose $\lambda(z)$ in this way.

Lemma 2 A solution of the equation

$$
\begin{gathered}
\psi(z)=\phi(z) \psi(z+1) \text { is } \\
\psi(z)-\prod_{i}^{k}\left(\frac{\Gamma\left(z+\beta_{i}\right)}{\Gamma\left(z+\beta_{i}\right)}\right) .
\end{gathered}
$$

Proof: Trivial since $\Gamma(z+1)=z \Gamma(z)$.

Lemma 3 Let $\mathrm{F} \mathrm{(z)} \mathrm{denote} \mathrm{the} \mathrm{series} \mathrm{on} \mathrm{the} \mathrm{LHS} \mathrm{of} \mathrm{(2).} \mathrm{Then}$ $F(z)$ converges uniformlv over compact subsets in the whole of the complex plane except in the neighbourhoods of $z=-\beta_{1},-\beta_{2}, \ldots,-\beta_{k} \cdot A l s o \psi(z)=F(z)$ is another solution of the equation mentioned in Lemma 1.

Proof: Follows from the facts that as $|z| \rightarrow \infty$ we have $\phi(z)=1-\frac{1}{z}+o\left(\frac{1}{|z|^{2}}\right)$ and $\lambda(z)=O\left(\frac{1}{|z|}\right)$. Because the $n^{\text {th }}$ term of the series for $F(z)$ is $O\left(\frac{1}{n}_{2 \leqslant m \leqslant n}^{\pi}\left(1-\frac{1}{m}+o\left(\frac{1}{m^{2}}\right)\right)\right)$ uniformly in $z$ so long as $z$ is in any compact set not containing the points $-\beta_{1},-\beta_{2}, \ldots,-\beta_{k}$. The last expression is

$$
o\left(\frac{1}{n} \operatorname{Exp}\left(-\sum_{2<m<n} \frac{1}{m}\right)\right)=O\left(\frac{1}{n^{2}}\right) .
$$




\section{Lemma 4 solution of the equation}

$$
\begin{gathered}
\psi(z)=\phi(z) \psi(z+1) \text { is } \\
\psi(z)=\mathrm{F}(z)-z+\prod_{i=1}^{k}\left(\frac{\left.\Gamma+\beta_{i}\right)}{\Gamma\left(z+\alpha_{i}\right)}\right) . \text { Moreover as }
\end{gathered}
$$

$z \rightarrow \infty$ through positive real values this solution is $O\left(z^{\varepsilon}\right)$ for every fixed $\boldsymbol{\varepsilon}>0$.

Proof: The first part follows from the fact that $\psi(z)=z$ and $F(z)$ are two solutions of the equation mentioned in lemma 1. If $A_{n}$ denotes the $n^{\text {th }}$ term in the series for $F(z)$, then

$$
\begin{aligned}
A_{n} & =O(\lambda(z+n) \phi(z) \phi(z+1) \ldots \phi(z+n)) \\
& =O\left(\frac{1}{z+n} \operatorname{Exp}\left(-\sum_{0<\gamma<n} \frac{1}{z+\gamma}\right)\right) \\
& \text { since } \sum_{0 \leqslant \gamma \leqslant n} \frac{1}{(z+\gamma)^{2}}=O(1), \\
& =O\left(\frac{1}{z+n} \operatorname{Exp}\left(-\sum_{1 \leqslant \gamma \leqslant n+z} \frac{1}{\gamma}+\sum_{\gamma \leqslant z+1} \frac{1}{\gamma}\right)\right) \\
& =O\left(\frac{z}{(z+n)^{2}}\right) .
\end{aligned}
$$

and so$$
\sum_{n=0}^{\infty} A_{n}=O\left(z_{\gamma>z-1} \gamma^{\frac{1}{2}}\right)=O(1) .
$$$$
\text { It now remains to check } \underset{i=1}{k}\left(\frac{\Gamma+\beta_{i}}{\Gamma\left(z+a_{i}\right.}\right)-z=O\left(z^{\varepsilon}\right) \text { as }
$$ 
$z \rightarrow \infty$. For this it suffices to prove that if $b_{i}$ and $d_{i} \quad(i=1$ to $k$ ) are complex numbers with $\Sigma b_{i}=\Sigma d_{i}$ then as $z \rightarrow \infty$, $\sum_{i=1}^{k}\left(\frac{\Gamma\left(z+b_{i}\right)}{\Gamma\left(z+d_{i}\right)}\right)=1+o\left(\frac{1}{z}\right)$, i.e. $\sum_{i=1}^{k} \log \left(\frac{\Gamma\left(z+b_{i}\right)}{\Gamma\left(z+d_{i}\right)}\right)$ $=O\left(\frac{1}{z}\right)$. This follows from the well-known asymptotic formula $\log \Gamma(z)=\left(z-\frac{1}{2}\right) \log z-z+\frac{1}{z} \log (2 \pi)+O\left(\frac{1}{z}\right)$, (as $z \rightarrow \infty)$.

\section{Lemma 5}

Let $z$ belong to some fired sub-interval of length $\frac{1}{10}$ of $(0, \infty)$ to the right of which $-\beta_{i}+1$ do not lie. Then the solution $\psi(z)$ of the equation in lemma 4 vanishes for all $z$ in the interval under question and hence identically for all $z$.

Proof: If $z$ belongs to the interval we have by lemma 4 ,

$\psi(z)=\phi(z) \psi(z+1)$

$$
\begin{aligned}
& =\phi(z) \phi(z+1) \ldots \phi(z+n) \psi(z+n+1) \\
& =O\left(\left(\operatorname{Exp}\left(-\sum_{1 \leqslant \gamma \leqslant n} \frac{1}{\gamma}\right)\right) n^{\dagger}\right)
\end{aligned}
$$

since $\phi(z) \phi(z+1) \ldots \phi(z+n)=O\left(\operatorname{Exp}\left(-\sum_{1 \leqslant \gamma \leqslant n} \frac{1}{\nu}\right)\right)$ and $(z+n+1)=O\left(n^{\frac{1}{2}}\right)$. Hence $(n$ being arbitrary) $\psi(z)$ is zero in the interval under question and hence for all $z$ by analytic continuation.

Lemma 5 completes the proof of Theorem 1.

\section{§ 3. Further Remarks}

We can allow $\Sigma \beta_{i}=j+\Sigma a_{i}$ where $j$ is any integer in Theorem 1 and state more general theorems. These and further 
generalizations will be reserved for another paper. Next we remark that it is perhaps possible to put all these in the more general set up of $q$ series, $q$-products and their limiting processes. With this in view we state the following theorem on basic $q$-series and products like $f(x)=\prod_{n=0}^{\infty}\left(1-x q^{n}\right)$ where $0<q<1$, and $x$ is a complex variable such that $f(x)$ and $(f(x))^{1}$ are both regular.

Theorem 2.

Let $i$ and $j$ run through numbers $i=1,2, \ldots, h_{1}, j=1,2$, $\ldots, h_{2}$ where $h_{1} \geqslant 0, h_{2}>0$ and $h_{1}+h_{2}>1$.

Put $\left.\mathrm{F}_{1}(x)=\left[\underset{i}{(\boldsymbol{\pi}} f\left(\boldsymbol{x} x_{i}\right)\right)\left(\underset{i}{\pi} f\left(x y_{j}\right)\right)^{-1}\right]$

where $x_{i}$ and $y_{j}$ are some fixed complex numbers. Then we have trivially $\mathrm{F}_{1}(x)=g(x) \mathrm{F}_{1}(x q)$ where $g(x)=\left(\underset{i}{\pi}\left(1-x x_{i}\right) \underset{i}{(\pi}\left(1-x y_{j}\right)\right)^{-1}$. Further if $\mu(x)=(g(x))^{-1}-1$ then $1-\mathrm{F}_{1}(x)=\mu(x) g(x)+\mu(x q) g(x) g(x q)$ $+\mu\left(x q^{2}\right) g(x) g(x q) g\left(x q^{2}\right)+\ldots \ldots$

whenever both sides make sense.

Proof: Denote the RHS in the second assertion by $\mathrm{F}_{2}(x)$. It is easy to check that both $1-\mathrm{F}_{1}(x)$ and $\mathrm{F}_{2}(x)$ satisfy the equation $\mathrm{J}(x)=\mu(x) g(x)+\mathrm{J}(x q) g(x)$ so that their difference $\mathrm{W}(x)=1-\mathrm{F}_{1}(x)-\mathrm{F}_{2}(x)$ satisfies,

$$
\mathrm{W}(\boldsymbol{x})=g(x) \mathrm{W}(x q) .
$$

But since $g(x) g(x q) g\left(x q^{2}\right) \ldots$ is convergent and $\mathrm{W}\left(x q^{m}\right)$ tends to zero as $n \rightarrow \infty$ the only solution of this equation is $\mathrm{W}(\boldsymbol{x}) \equiv 0$, and this proves the theorem. 
Remark 1. Let $x_{1}-x_{2}=\ldots x_{h_{1}}=1$. Then $g(x)=\left(\frac{{ }_{i}^{\left(1-x y_{j}\right)}}{(1-x)^{h_{1}}}\right)^{-1}$ and

$\mu(x)=(g(x))^{-1}-1=\frac{\pi\left(1-x x_{j}\right)-(1-x)^{h_{1}}}{(1-x)^{h_{1}}}$

$=\frac{\left(-x \sigma_{1}+x^{2} \sigma_{2}-\ldots\right)-\left(-\left(_{1}^{h_{1}}\right) x+\left(_{2}^{h_{1}}\right) x^{2}-\ldots\right)}{(1-x)}$

$=\sum(1-x)^{\gamma} a_{\gamma}^{\prime}$

$-h_{1} \leqslant \gamma \leqslant\left|h_{1}-h_{2}\right|$

where $a_{\gamma}$ are polynomials in $y_{j}$. We thus obtain an expression

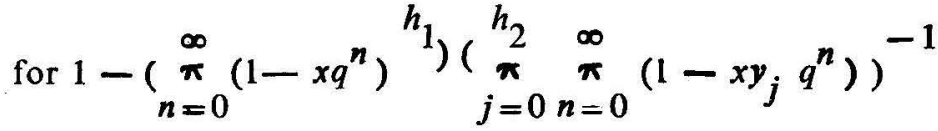
in terms of $g(x), g(x q), \ldots$ where $g(x)$ is already described.

\section{§4. Concluding Remarks.}

I have other results on series integrals and continued fractions. ( 1 hope to publish them in a continuation of the present series). Here I take the opportunity of mentioning some of the results (for some of them see [3]) which I read at the Indian Science Congress 45th Session, Madras (1958), when the then President (of the Mathematics Section) Professor B. S. Madhava Rao asked me to present my results. 


\section{Theorem 3.}

$$
\begin{aligned}
& \text { Let } \quad \mathrm{H}_{n} \approx 1+\frac{1}{2}+\frac{1}{3}+\ldots+\frac{1}{n}(n>1) . \text { Then } \\
& \sum_{n=1}^{\infty} 2^{-n} \mathrm{H}_{n}^{3}=\zeta(3)+\frac{1}{3}\left\{\pi^{2} \log 2+(\log 2)^{3}\right\} \text {, } \\
& \log _{8}(\pi)_{n=1}^{\infty}(-1)^{n} \frac{\mathrm{H}_{n}^{3}}{n}=\frac{9}{8} \zeta(3) \log 2+ \\
& \frac{1}{4}(\log 2)^{4}-\frac{1}{8}(\pi \log 2)^{2}-\frac{\pi^{4}}{144}, \\
& \sum_{n=1}^{\infty}(-1)^{n}(3 n+1) 2^{-n} \mathrm{H}_{n}^{3}=(\log 3-\log 2)^{2} \text {, } \\
& \sum_{n=1}^{\infty} \frac{\mathrm{H}_{n}^{3}}{n(n+1)}-\frac{\pi^{4}}{9} \text {, } \\
& \sum_{n=1}^{\infty} n 2^{-n-1}{ }_{n}^{4}=\frac{15}{4} \zeta(3)+\frac{13}{6} n^{2} \log 2+\frac{7}{3}(\log 2)^{3} \text {. }
\end{aligned}
$$

The proofs of these and others involving higher powers $\mathrm{H}_{n}^{k}(k=1,2,3, \ldots)$ will appear elsewhere.

Professor B. S. Madhava Rao will be 80 years old in 1980. On the occasion of his eightieth birthday I take the opportunity of paying my regards to him and expressing my indebtedness to him.

MANUSCRIPT COMPLETED ON 14 FEBRUARY 1980.

School of Mathematics

Tata Institute of Fundamental Research

Homi bhabha Road, Bombay 400005.

INDIA. 


\section{References}

(1) L. Euler, De Fractionibus continuis OPERA OMNIA, Volume VIIl, p. 371 (362-390); Plenior expositio serierum illarum memorabilium quae ex unciis potestatum binonii formantur, ibid Volume XVI, Sectio Prima p. 233 (193 - 234).

(2) G. H. Hardy, Ramanujan (Twelve lectures on subjects suggested by his life and work), Chelsea Publishing Company, New York, N. Y. (1940).

(3) K. Ramachandra, On the summation of certain series, involving $H_{n}=1+\frac{1}{2}+\cdots+\frac{1}{n}$; Proceedings of the Forty-fifth Indian Science Congress, Madras (1958), Maths abstract no. 3, page 1. 